\title{
Phase Registration of a Single Quasi-Periodic Signal Using Self Dynamic Time Warping
}

\author{
Yasushi Makihara ${ }^{1}$, Ngo Thanh Trung ${ }^{1}$, Hajime Nagahara ${ }^{2}$, \\ Ryusuke Sagawa $^{3}$, Yasuhiro Mukaigawa ${ }^{1}$, and Yasushi Yagi ${ }^{1}$ \\ 1 Osaka University, 8-1 Mihogaoka, Ibaraki, Osaka, 567-0047, Japan \\ 2 Kyushu University, 744, Motooka, Nishiku, Fukuoka, 819-0395, Japan \\ 3 AIST, 1-1-1 Umezono, Tsukuba, Ibaraki, 305-8568, Japan
}

\begin{abstract}
This paper proposes a method for phase registration of a single non-parametric quasi-periodic signal. After a short-term period has been detected for each sample by normalized autocorrelation, Self Dynamic Time Warping (Self DTW) between a quasi-periodic signal and that with multiple-period shifts is applied to obtain corresponding samples of the same phase. A phase sequence is finally estimated by the optimization framework including the data term derived from the correspondences, the regularization term derived from short-term periods, and a monotonic increasing constraint of the phase. Experiments on quasiperiodic signals from both simulated and real data show the effectiveness of the proposed method.
\end{abstract}

\section{Introduction}

Periodic signal analysis has been widely studied in the computer vision field as well as signal processing field, as the periodic signal plays quite an important role in many applications ranging from transmitting information via a radio carrier wave [1] [2] in the electronic communication field to periodic motion detection from video, periodic action recognition (e.g., walking and running), person identification from periodic motion (e.g., gait-based person identification [3]).

Such a periodic signal is often modulated in terms of amplitude, frequency, and phase by design or by chance, and is converted into a quasi-periodic signal. Typical examples of intentional modulation are Amplitude Modulation (AM) and Frequency Modulation (FM) [1] used in radio broadcasts, and Phase Modulation (PM) [2] used in radio control, where a carrier wave with known parameters is given as reference and the modulation is estimated based on the carrier wave.

On the other hand, accidental modulation is induced by a fluctuation in the sampling interval (network camera with limited communication band width) or that of the periodic signal source itself (e.g., fluctuations in human walking patterns). Estimating phases from such phase-modulated quasi-periodic signals is quite an important task in many applications. For example, temporal interpolation of a video with constant phase evolution needs the correct phase information for each key frame. Moreover, temporal super resolution of a periodic image sequence needs accurate phase registration data with sub-sampling order displacement of phase, in the same way that spatial super resolution needs image registration data with sub-pixel order displacement [4]. Phase registration data is also essential to reconstruct a manifold parameterized by phase in 
periodic action analysis and recognition and accurate period segmentation for periodic signal matching. In cases where a reference periodic signal is available, Dynamic Time Warping (DTW) [5] (more specifically, continuous DP [6] in the periodic signal case) is a powerful tool for matching two sequences with non-linear time warping, in the sense that matching results give phase registration data. The reference signal is, however, usually not available in the above applications.

This paper tackles the challenging problem of phase registration from a single quasi-periodic signal. After a short-term period has been detected for each sample, Self DTW between the quasi-periodic signal and that with multiple-period shifts is applied to obtain corresponding samples with the same phase. A phase sequence is finally estimated in a sub-sampling order by the optimization framework where an objective function is composed of the data term derived from the correspondences, the regularization term derived from short-term periods, and a monotonic increasing constraint of the phase.

\section{Related work}

Parametric representation: A periodic signal is usually represented by a periodic function parameterized by amplitude, frequency, and phase, and it is often observed together with additive noise. Such parametric expression is widely used in the context of periodic signal reconstruction [7] and detection [8], enhancement of a specific frequency [9], estimation of amplitude [10], and decomposition of multiple periodic signals [11] [12] [13]. The common key technique in these approaches is parameter estimation and hence, non-parametric periodic signals are out of scope.

Linear time warping: Linear time warping is conventionally used in periodic action recognition such as gait recognition [14] [15] [16]. Periods are usually first detected as an interval of signal peaks [3] by maximum entropy spectrum estimation [17] or by maximum normalized autocorrelation [18]. The signals are then linearly stretched/shrunk so that the periods of two signals match. Naturally, these methods cannot deal with non-linear time warping within a period.

Non-linear time warping: Dynamic Time Warping (DTW) [5] has been widely used for elastic matching of two sequences in the field of action recognition [19] and gait recognition [20]. The Hidden Markov Model (HMM) is a probabilistic framework version of the DTW, which is also used in phase state estimation in walker motion extraction [21], gait silhouette refinement [22] [23], and gait recognition [24] [25]. An HMM needs sufficient training sequences and hence, cannot be applied directly to phase registration from a single sequence. Moreover, the number of states should be sufficiently large to realize a sub-sampling order phase estimation and this leads to an explosive increase in the number of training samples required.

\section{Phase registration}

\subsection{Problem statement}

Given a periodic function of the multi-dimensional signal $\boldsymbol{f}(t)$ with period $P$ that satisfies $\boldsymbol{f}(t+j P)=\boldsymbol{f}(t) \forall j \in \mathbb{Z}$, a time normalized by period $P$, is introduced as an absolute phase $s$ and a relative phase $\tilde{s}$ as 


$$
\begin{aligned}
& s=s_{P}(t)=\frac{1}{P} t \\
& \tilde{s}=s-\lfloor s\rfloor,
\end{aligned}
$$

where $s_{P}(t)$ is a phase function, and $\lfloor s\rfloor$ is a floor function. A normalized periodic function is subsequently introduced as

$$
\boldsymbol{h}(s)=\boldsymbol{f}\left(s_{P}^{-1}(s)\right)
$$

which satisfies $\boldsymbol{h}(s)=\boldsymbol{h}(\tilde{s}) \forall s$.

Next, it is assumed that the phase function $s_{P}(t)$ is distorted by fluctuation into $s_{Q}(t)$ and that the periodic signal $\boldsymbol{f}(t)$ is converted to a quasi-periodic signal $\boldsymbol{g}(t)$, which is subject to

$$
\boldsymbol{g}(t)=\boldsymbol{h}\left(s_{Q}(t)\right)=\boldsymbol{f}\left(s_{Q}\left(s_{P}^{-1}(s)\right)\right) .
$$

Given the quasi-periodic signal $\boldsymbol{g}(t)$ and its phase function $s_{Q}(t)$, the periodic function is reconstructed as

$$
\boldsymbol{h}(s)=\boldsymbol{g}\left(s_{Q}^{-1}(s)\right) .
$$

In addition, since the signal is usually quantized in observation, we redefine the above variables at quantized time $t_{i}(i=0, \ldots, N)$ with subscription $i$ (e.g., $\left.\boldsymbol{g}_{i}=\boldsymbol{g}\left(t_{i}\right)\right)$. Therefore, our objective is to estimate a phase sequence $\boldsymbol{S}_{Q}=\left\{s_{Q, i}\right\}$ from a given quasi periodic sequence $\boldsymbol{G}=\left\{\boldsymbol{g}_{i}\right\}$. This is referred to as the phase registration problem in this paper.

On the other hand, the following ambiguity of the phase function and normalized periodic function remains. Given another phase function $s_{Q}^{\prime}(t)$ and another normalized periodic function $\boldsymbol{h}^{\prime}(s)=\boldsymbol{h}\left(s_{Q}^{\prime}\left(s_{Q}^{-1}(s)\right)\right)$ that satisfies $\boldsymbol{h}^{\prime}(s)=\boldsymbol{h}^{\prime}(\tilde{s}) \forall s$, another quasi-periodic function $\boldsymbol{g}^{\prime}(t)$ is defined in two ways as $\boldsymbol{g}^{\prime}(t)=\boldsymbol{h}\left(s_{Q}^{\prime}(t)\right)=\boldsymbol{h}^{\prime}\left(s_{Q}(t)\right)$. Therefore, given the quasi-periodic function $\boldsymbol{g}(t)$, the ambiguity of combinations of the phase function $s_{Q}(t)$ and the normalized periodic function $\boldsymbol{h}(s)$ remains. In this paper, we estimate one of the phase functions.

\subsection{Pseudo period estimation}

First, we define a differential of the phase function

$$
\frac{d s_{Q}(t)}{d t}=\frac{1}{P_{Q}(t)}
$$

where $P_{Q}(t)$ is called the pseudo period in this paper. Note that the pseudo period $P_{Q}(t)$ is equivalent to the period $P$ for the periodic signal, which is obvious from Eq. (2). The equation in quantized domain is also defined as

$$
s_{Q, i+1}-s_{Q, i}=\frac{1}{P_{Q, i}} .
$$

Then, the pseudo period is estimated by maximizing a short-term normalized autocorrelation as 


$$
\begin{aligned}
\hat{P}_{Q, i} & =\arg \max _{P_{Q} \in\left[P_{\text {min }}, P_{\text {max }}\right]} C_{i}\left(P_{Q}\right) \\
C_{i}\left(P_{Q}\right) & =\frac{\sum_{\tau \in I_{i}} \boldsymbol{g}_{\tau}^{T} \boldsymbol{g}_{\tau+P_{Q}}}{\sqrt{\sum_{\tau \in I_{i}}\left\|\boldsymbol{g}_{\tau}\right\|^{2}} \sqrt{\sum_{\tau \in I_{i}}\left\|\boldsymbol{g}_{\tau+P_{Q}}\right\|^{2}}} \\
I_{i} & =\left\{\tau \mid i-\alpha P_{\text {max }} \leq \tau \leq i+\alpha P_{\text {max }}, \tau \in \mathbb{Z}\right\},
\end{aligned}
$$

where $\left[P_{\min }, P_{\max }\right]$ is a domain of the pseudo period which is obtained by existing methods of period detection or given by prior knowledge, and $\alpha$ is a coefficient to control the size of the window function for the short-term mask.

\subsection{Self Dynamic Time Warping}

Given a correspondence of two samples $i$ and $u_{i}^{j}$ with $j$ periods difference (call this the $j$ th period correspondence and denote it as $\boldsymbol{x}=[i, u]$ ), they are ideally subject to

$$
\begin{gathered}
s_{Q, u_{i}^{j}}-s_{Q, i}=j \\
\boldsymbol{g}_{u_{i}^{j}}=\boldsymbol{g}_{i},
\end{gathered}
$$

where the equations denote the phase constraint and signal consistency, respectively. Hence, Eq. (11) is exploited as a constraint for phase registration, and we try to find the correspondences based on the signal consistency of Eq. (12) by applying Self Dynamic Time Warping (Self DTW) to the quasi periodic sequence $\boldsymbol{G}$.

First, an initial estimate of the $j$ th period correspondence $\hat{\boldsymbol{x}}_{i}^{j}=\left[i, \hat{u}_{i}^{j}\right]$ is obtained from the estimated pseudo period $\hat{P}_{Q, i}$ in a recursive manner as

$$
\hat{u}_{i}^{j}=\hat{u}_{i}^{j-1}+\hat{P}_{Q, \hat{u}_{i}^{j-1}}, \hat{u}_{i}^{0}=i
$$

Second, lower and upper bounds of the $j$ th period correspondence are set to

$$
\begin{aligned}
u_{\text {low }, i}^{j} & =\max \left\{\hat{u}_{i}^{j}-\beta \hat{P}_{Q, \hat{u}_{i}^{j}}, 0\right\} \\
u_{u p, i}^{j} & =\min \left\{\hat{u}_{i}^{j}+\beta \hat{P}_{Q, \hat{u}_{i}^{j}}, N\right\} .
\end{aligned}
$$

Thus, a Self DTW path search region is defined as $R^{j}=\left\{\boldsymbol{x}=[i, u] \mid \hat{u}_{l o w, i}^{j} \leq u \leq\right.$ $\left.\hat{u}_{u p, i} \forall i \in[0, N]\right\}$, and subsequently the source and terminal regions are set to $R_{S}^{j}=$ $\left\{\boldsymbol{x}=[0, u] \mid \boldsymbol{x} \in R^{j}\right\}$ and $R_{T}^{j}=\left\{\boldsymbol{x}=[i, N] \mid \boldsymbol{x} \in R^{j}\right\}$, respectively, as illustrated in Fig. 1. Now, the correspondence problem is decoded as continuous dynamic programming [6] in the search region $R^{j}$.

The formulation is given as follows. A cumulative cost $c(\boldsymbol{x})$ and a counter $n(\boldsymbol{x})$ are introduced and these are initialized for $\boldsymbol{x} \in R_{S}^{j}$ as

$$
c(\boldsymbol{x})=c_{I}(\boldsymbol{x}), n(\boldsymbol{x})=1,
$$

where $c_{I}(\boldsymbol{x})$ is a cost function for the signal intensity difference given as $c_{I}(\boldsymbol{x})=$ $\left\|\boldsymbol{g}_{i}-\boldsymbol{g}_{u}\right\|$.

Next, a transition process is considered. We limit the previous state $\boldsymbol{x}_{p}$ to the current state $\boldsymbol{x}$ to $T^{j}(\boldsymbol{x})=\{[i-1, u-1],[i-2, u-1],[i-1, u-2]\} \cap R^{j}$ and define the optimal previous state to the current state as $\boldsymbol{x}_{p}^{j *}(\boldsymbol{x})$, which is given as

$$
\boldsymbol{x}_{p}^{j^{*}}(\boldsymbol{x})=\arg \min _{\boldsymbol{x}_{p} \in T^{j}(\boldsymbol{x})}\left\{\frac{c\left(\boldsymbol{x}_{p}\right)}{n\left(\boldsymbol{x}_{p}\right)}+c_{T}\left(\boldsymbol{x}, \boldsymbol{x}_{p}\right)\right\},
$$




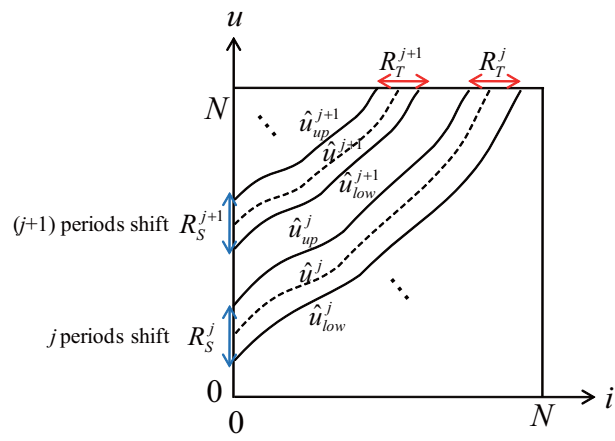

Fig. 1: Overview of Self DTW

where the first and second terms on the right side are, respectively, the counternormalized previous cumulative cost and the transition cost function, given as $c_{T}\left(\boldsymbol{x}, \boldsymbol{x}_{p}\right)=$ $\left\|\boldsymbol{x}-\boldsymbol{x}_{p}\right\|_{L_{1}}$. Then, the cumulative cost and the counter are updated as

$$
\begin{aligned}
c(\boldsymbol{x}) & =c\left(\boldsymbol{x}_{p}^{j}(\boldsymbol{x})^{*}\right)+c_{I}(\boldsymbol{x})+c_{T}\left(\boldsymbol{x}, \boldsymbol{x}_{p}^{j^{*}}(\boldsymbol{x})\right) \\
n(\boldsymbol{x}) & =n\left(\boldsymbol{x}_{p}^{j *}(\boldsymbol{x})\right)+1
\end{aligned}
$$

After the cost propagation of all the states in $R^{j}$, the optimal state at the terminal is

$$
\boldsymbol{x}_{T}^{j *}=\arg \min _{\boldsymbol{x} \in R_{T}^{j}} \frac{c(\boldsymbol{x})}{n(\boldsymbol{x})} .
$$

Subsequently, the terminal counter and the optimal terminal state are redefined, respectively, as $n^{j}=n\left(\boldsymbol{x}_{T}^{j *}\right)$ and $\boldsymbol{x}_{n_{j}}{ }^{*}=\boldsymbol{x}_{T}^{j}{ }^{*}$ for convenience, and the optimal path is back tracked as $\boldsymbol{x}_{i}^{j^{*}}=\boldsymbol{x}_{p}^{j *}\left(\boldsymbol{x}_{i+1}^{j}{ }^{*}\right)$ for $i=n^{j}-1, \ldots, 1$. In the following sections, the optimal correspondence sequence is denoted as $\boldsymbol{X}^{j}=\left\{\boldsymbol{x}_{i}^{j} \mid i=1, \ldots, n^{j}\right\}$.

\subsection{Phase sequence optimization}

Phase sequence $\boldsymbol{S}_{Q}$ is estimated by taking the following three points into consideration: (1) the obtained optimal correspondence sequence $\boldsymbol{X}^{j},(2)$ the smoothness of the phase sequence $\boldsymbol{S}_{Q}$, and (3) monotonically increasing the phase sequence $\boldsymbol{S}_{Q}$ as

$$
\begin{aligned}
& \boldsymbol{S}_{Q}^{*}= \arg \min _{\boldsymbol{S}_{Q}} D\left(\boldsymbol{S}_{Q}\right) \\
& D\left(\boldsymbol{S}_{Q}\right)= \sum_{j} \sum_{[i, u] \in \boldsymbol{X}^{j}}\left(s_{Q, u}-s_{Q, i}-j\right)^{2} \\
&+\lambda \sum_{i=0}^{N-1}\left(s_{Q, i+1}-s_{Q, i}-\frac{1}{\hat{P}_{Q, i}}\right)^{2} \\
& \text { subject to } s_{Q, i+1}-s_{Q, i} \geq 0 \forall i=0, \ldots, N-1,
\end{aligned}
$$

where the first and second terms on the right side of Eq. (22) are the data term derived from the correspondences and the regularization term derived from Eqs. (7) and (8), respectively, and $\lambda$ is the regularization term coefficient. 
As described before, the ambiguity of the phase function remains. First, a constant shift $\Delta s$ in $s_{Q}^{\prime}(t)=s_{Q}(t)+\Delta s$ does not change the value of the objective functions and the constraints at all, because all the $s_{Q, i}$ are used in the subtraction form. Therefore, the following constraint is added without loss of generality

$$
s_{Q, 0}=0
$$

Second, considering another phase function $s_{Q}^{\prime}(t)=s_{Q}(t)+r(t)$ with a quasiperiodic shift $r(t)$ that satisfies

$$
\begin{aligned}
r(t) & =r\left(t^{\prime}\right) \forall\left[t, t^{\prime}\right] \in\left\{\left[t, t^{\prime}\right] \mid \tilde{s}_{Q}\left(t^{\prime}\right)=\tilde{s}_{Q}(t)\right\} \\
\frac{d r(t)}{d t} & \geq-\frac{d s_{Q}(t)}{d t}
\end{aligned}
$$

the quasi-periodic shift $r(t)$ does not change the data term of the objective functions assuming no correspondence error. In other words, the quasi-periodic shift $r(t)$ depends on a tradeoff between the correspondence errors in the data term and residuals between inverses of the correct pseudo period $P_{Q, i}$ and its estimate $\hat{P}_{Q, i}$ in the regularization term.

Finally, because the objective function $D\left(\boldsymbol{S}_{Q}\right)$ is a quadratic form and the constraints of Eqs. (23) and (24) are a linear form, the above optimization problem is solved by convex quadratic programming using the active set method.

\section{Experiments}

\subsection{Simulation data}

We carried out experiments on simulation data to confirm the effectiveness of the proposed phase registration. First, we generated three normalized periodic functions with a single dimension as a non-parametric function, with the second order differential $\left(d^{2} h / d s^{2}\right)$ randomly drawn from a uniform distribution in the domain [-500,500] and with boundary conditions $h(1)=h(0)=0$. The phase function $s_{Q}(t)$ was also generated by a non-parametric scheme in the same way. Given the pseudo period function $P_{Q}(t)$ with second order differential $\left(d^{2} P_{Q} / d t^{2}\right)$ drawn from a uniform distribution in the domain $[-0.25,0.25]$ with boundary conditions $P_{Q}(0)=P_{Q}(T)=P$, where $T$ is the time at the final frame and $P$ is a predefined period, the phase function $s_{Q}(t)$ is given by the first order differential equation $d s_{Q} / d t=1 / P_{Q}(t)$ with initial condition $s_{Q}(t)=0$. In this simulation, $T$ and $P$ were set to 10 and 100 , respectively.

Third, quasi-periodic sequences were generated by sampling at $(1 / P)$ intervals as $g_{i}=h\left(s_{Q}(i t / P)\right), i=0, \ldots, N$, where $N=T P$ is the sample ID at the final frames. Fourth, sequences with noise were also generated as $g_{i}^{\prime}=g_{i}+\delta$, where $\delta$ is drawn from a Gaussian distribution with standard deviation $\sigma=0.1$. The other parameters used in each process were set experimentally as $\alpha=1.0, \beta=0.3$, and $\lambda=10.0$. The generated signals were phase-modulated as shown in Fig. 2(a).

If a reference signal is not given in the problem statement, existing methods such as continuous cyclic DP and cyclic HMM cannot be applied. Therefore, we regard the following scheme based on the estimated pseudo period with Short-Term Period Detection (STPD) as a baseline algorithm for comparison:

$$
s_{Q, i+1}=s_{Q, i}+\hat{P}_{Q, i},
$$




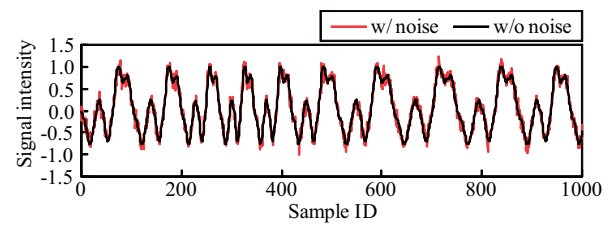

(a) Input quasi-periodic sequence

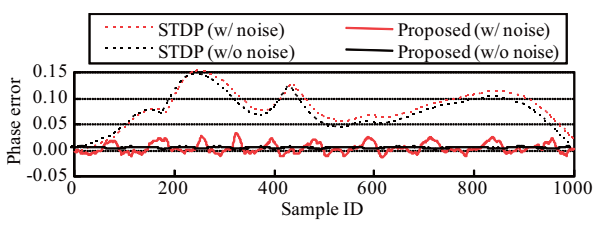

(b) Phase error

Fig. 2: Quasi-periodic input sequence and its phase error

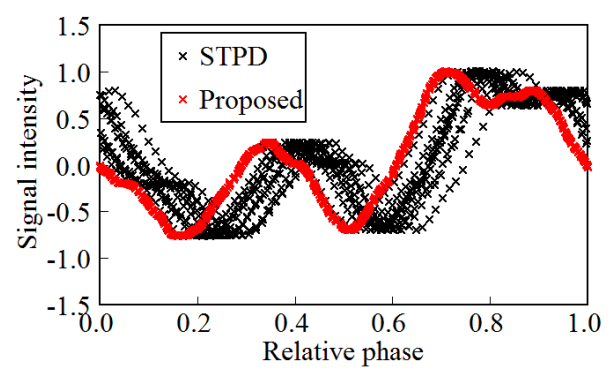

(a) Without noise

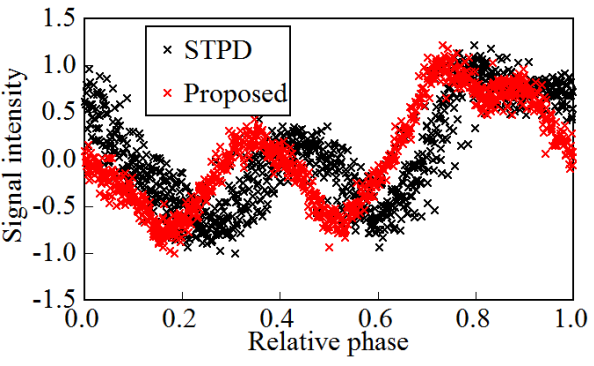

(b) With noise

Fig. 3: Phase registration results for simulated data

where we initialize $s_{Q, 0}=0$. Note that this is also equivalent to the case in which the regularization coefficient $\lambda$ is set to infinity in the proposed framework.

First, we evaluated the errors between the estimated phase and the ground truth in Fig. 2(b). Because the ambiguity of the phase function is as described previously, bias components in the errors should be ignored here. As a result, the error variance in noisy data is larger than that in data without noise in the proposed method. The error patterns are, however, still similar to a quasi-periodic form; this implies the possibility of another combination of the phase function $s_{Q}^{\prime}(t)$ and the normalized periodic function $h^{\prime}(s)$. On the other hand, the error variance in the baseline method (STPD) is larger than that in the proposed method, and furthermore, the error patterns are not similar to a quasi-periodic form.

Next, phase registration results were evaluated in the domain of the relative phase $\tilde{s}_{Q, i}^{*}$ and the corresponding signal intensity $g_{i}$ in Fig. 3. Note that their plots form a certain normalized periodic function $h(s)$ if the phase is correctly registered. As a result, the plots for the data without noise in the proposed method lie on a single curve and they form similar curves to the original signals (Fig. 3(a)). Moreover, the plots for the noisy data seem to lie within the range of additive noise distribution in the quasiperiodic sequence (Fig. 3(b)). On the other hand, the plots of the baseline method are widely distributed around the original signals due to incorrect phase registration.

\subsection{Real data}

We also conducted an experiment on a gait silhouette sequence with gradual speed variations ranging from $6 \mathrm{~km} / \mathrm{h}$ to $10 \mathrm{~km} / \mathrm{h}$ as shown in Fig. 4 . An image sequence of a walking person on a speed-controllable treadmill was captured at $60 \mathrm{fps}$ and a 


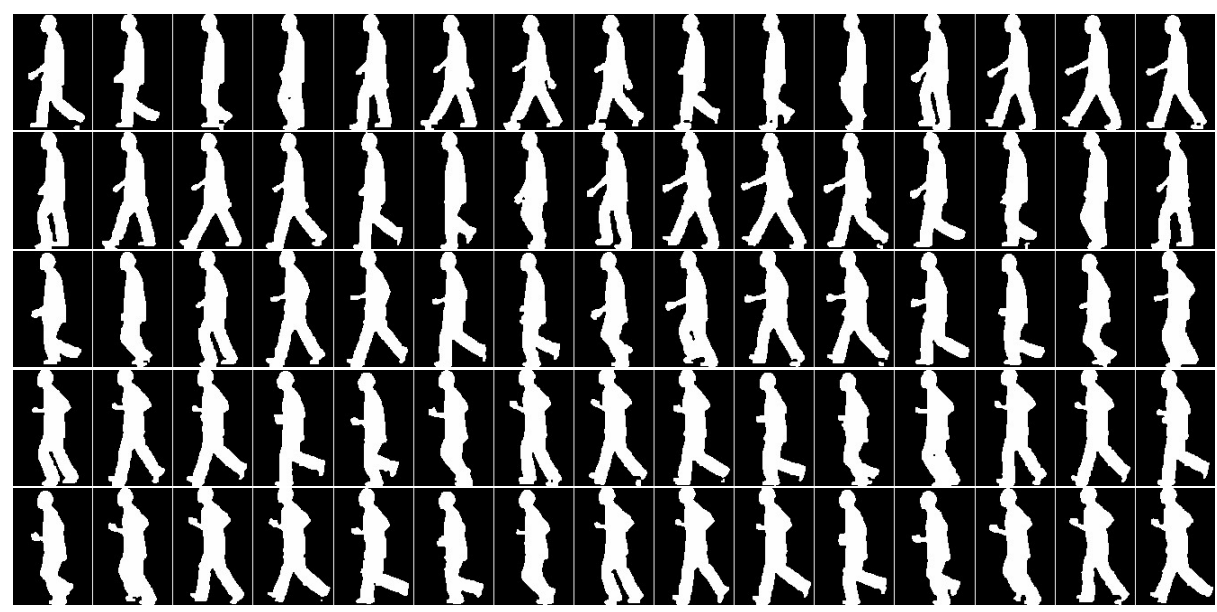

Fig. 4: Subsequences of input gait silhouettes (every 4 frames). Top to bottom rows correspond to $6,7,8,9$, and $10 \mathrm{~km} / \mathrm{h}$, respectively. Note that the phases among different walking speeds are not synchronized.

size-normalized silhouette sequence ( 88 by 128 pixels) extracted by graph cut-based segmentation in conjunction with background subtraction [26]. PCA was then applied to the silhouette images and subsequently, the proposed method was applied to the dimension-reduced data.

Figure 5 shows gait silhouette images aligned at the estimated relative phase. Despite the significant variation in gait style due to large speed variations from walking $(6 \mathrm{~km} / \mathrm{h})$ to running $(10 \mathrm{~km} / \mathrm{h})$, all the gait phases, such as double-support phase and single-support phase, are well registered for the different speeds. Note that nonuniform alignment intervals of the gait silhouette images in Fig. 5 represent non-linear time distortion due to the gait fluctuation obtained by the proposed Self DTW.

From an application viewpoint, phase-registered image sequences are quite useful. For example, given just a single walking sequence with speed variation, a gait manifold parameterized by both phase and walking speed can be constructed by re-sampling the phase-registered speed-varied gait image sequence as shown in Fig. 6. The gait manifold enables us to analyze the gait pose transition by walking speed for the same phase as well as that by phase for the same walking speed. Moreover, in the context of gait recognition with speed variations, the $2 \mathrm{D}$ gait manifold is provided as an efficient gallery expression, unlike the existing $1 \mathrm{D}$ gait manifold parameterized only by phase [16]. A set of $1 \mathrm{D}$ gait manifolds with different speeds depicted as colored loops in Fig. 6 cannot deal with variations in walking speed within a period, particularly as they do not provide phase registration information for different walking speeds depicted as gray lines in Fig. 6. On the other hand, since a 2D gait manifold has such phase registration information for different walking speeds, it can appropriately match a sequence with walking speed variations within a period in the framework of 1D-2D (input to gallery) dynamic programming. Note that the proposed method is applicable not only to gait with speed variation, but also to general quasi-periodic signals undergoing transition 


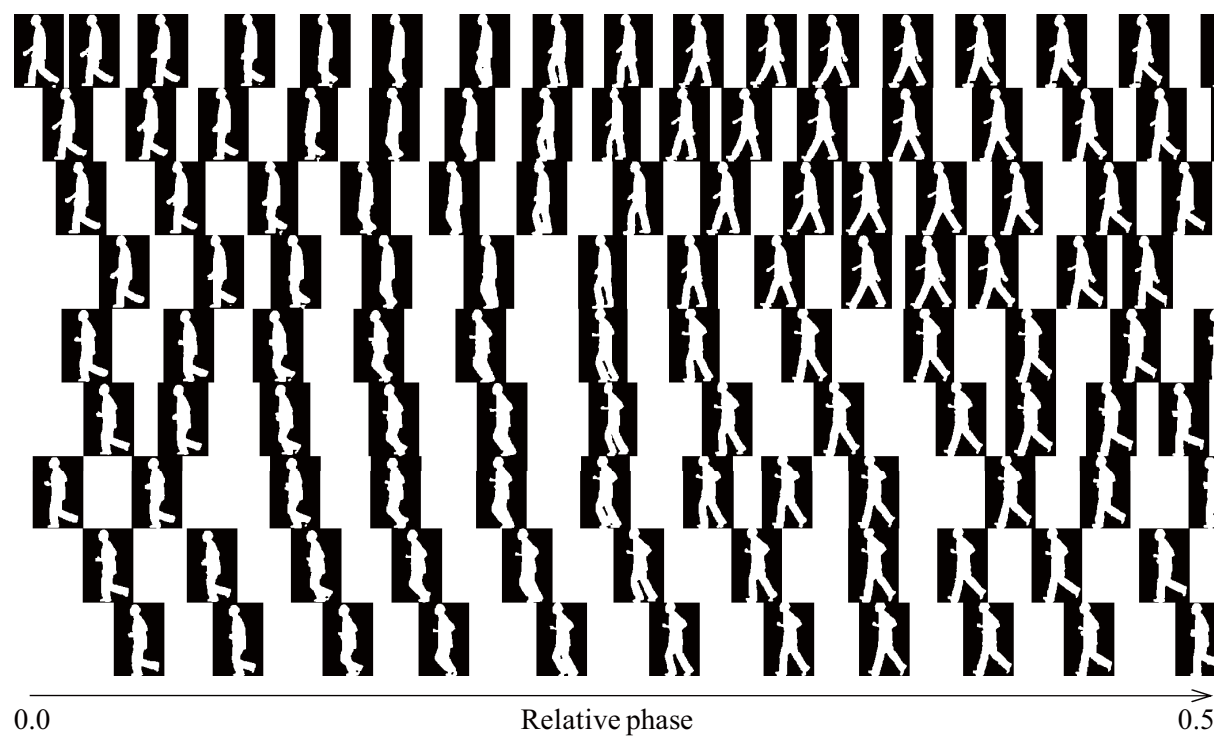

Fig. 5: Gait silhouette images aligned at the estimated phases (every 2 frames, a half gait period). The horizontal axis indicates the relative phase $\tilde{s}$ and each silhouette image is aligned at the estimated relative phase. The vertical axis indicates the number of periods (every 5 periods). Changes in the rows from top to bottom represent a gradual speed increase from $6 \mathrm{~km} / \mathrm{h}$ to $10 \mathrm{~km} / \mathrm{h}$.

by factors other than phase, such as periodic action recognition with gradual view changes or periodic signal analysis with gradual attenuation ${ }^{4}$.

\section{Conclusion}

This paper proposed a method for phase registration of a single non-parametric quasiperiodic signal. Having detected a short-term period for each sample by normalized autocorrelation, correspondences of multiple-period shifts are obtained by Self Dynamic Time Warping (Self DTW), which are used in the subsequent phase optimization framework.

Future works include eliminating ambiguity between the phase function and normalized period function based on the periodicity of the estimated phase sequence, extension of the proposed method for a quasi-periodic signal with both phase and amplitude modulation, and application to matching and time super-resolution of the quasi-periodic signals.

Acknowledgement. This work was supported by Grant-in-Aid for Scientific Re$\operatorname{search}(\mathrm{S}) 21220003$.

\footnotetext{
${ }^{4}$ In these cases, the manifold is parameterized by phase and view or degree of attenuation.
} 


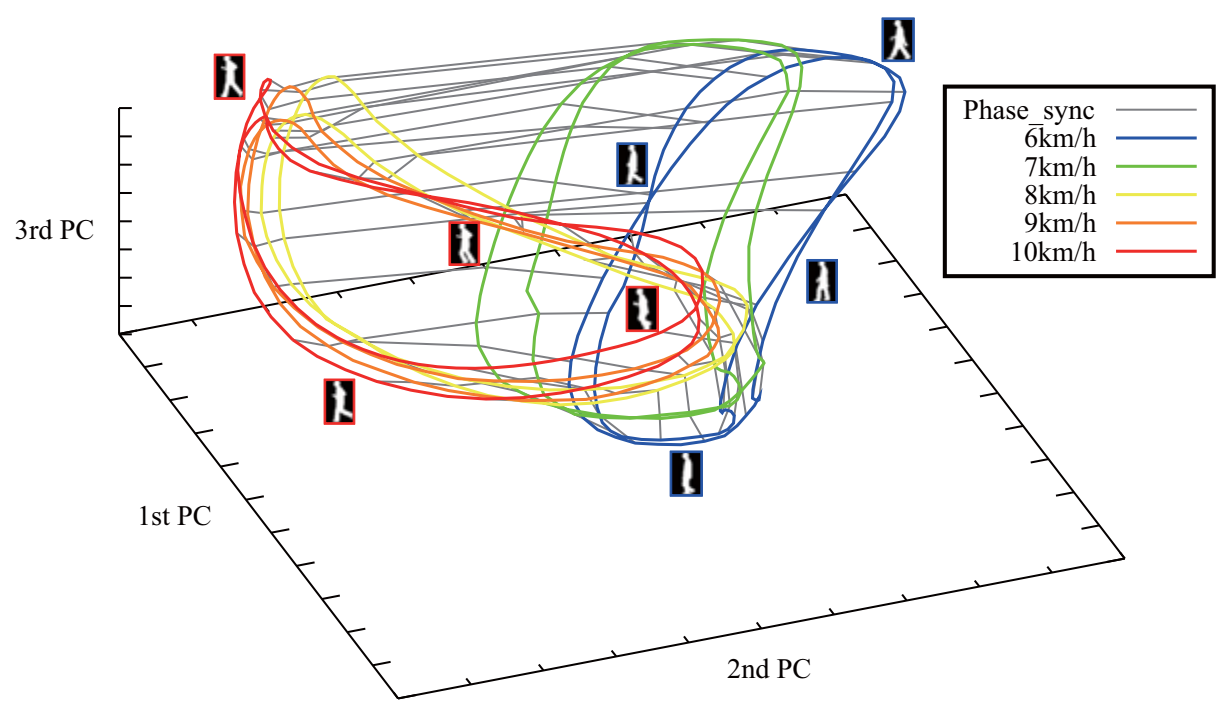

Fig. 6: A 2D gait manifold parameterized by phase and walking speed. While each color loop depicts a manifold for each walking speed parameterized by phase, gray lines represent phase synchronization among the walking speeds.

\section{References}

1. Newkirk, D., Karlquist, R.: Communication systems, 2nd edition. McGraw-Hill, Inc (1981)

2. Anderson, J.B., Aulin, T., Sundberg, C.E.: Digital phase modulation. Springer (1986)

3. Sarkar, S., Phillips, J., Liu, Z., Vega, I., Grother, P., Bowyer, K.: The humanid gait challenge problem: Data sets, performance, and analysis. Trans. of Pattern Analysis and Machine Intelligence 27 (2005) 162-177

4. van Ouwerkerk, J.: Image super-resolution survey. Image and Vision Computing 24 (2006) 1039-1052

5. Sakoe, H., Chiba, S.: Dynamic programming algorithm optimization for spoken word recognition. IEEE Transactions on Acoustics, Speech and Signal Processing 26 (1978) 43-49

6. Oka, R.: Spotting method for classification of real world data. Computer Journal 41 (1998) 559-565

7. Aronsson, D., Bjornemo, E., Johansson, M.: Estimation and detection of a periodic signal. In: Proc. of American Institute of Physics Conference, Bayesian Inference and Maximum Entropy Methods In Science and Engineering. Volume 872. (2006) 139-146 
8. Znak, V.: Some aspects of estimating the detection rate of a periodic signal in noisy data and the time position of its components. Pattern Recognition and Image Analysis 19 (2009) 539-545

9. Handel, P., Tichavsky, P.: Adaptive estimation for periodic signal enhancement and tracking. International Journal of Adaptive Control and Signal Processing 8 (2007) 447-456

10. BARROS, A.K., OHNISHI, N.: Amplitude estimation of quasi-periodic physiological signals by wavelets. IEICE transactions on information and systems E83-D (2000) 2193-2195

11. Gruber, P., Todtli, J.: Estimation of quasiperiodic signal parameters by means of dynamicsignal models. IEEE Transactions on Signal Processing 42 (1994) 552-562

12. NAKASHIZUKA, M.: A sparse decomposition method for periodic signal mixtures. IEICE Transactions on Fundamentals of Electronics, Communications and Computer Sciences E91-A (2008) 791-800

13. Wong, H., Sethares, W.A.: Estimation of pseudo-periodic signals. In: Proc. of IEEE Int. Conf. on Acoustics, Speech, and Signal Processing. Volume 2. (2004) $557-560$

14. Murase, H., Sakai, R.: Moving object recognition in eigenspace representation: Gait analysis and lip reading. Pattern Recognition Letters 17 (1996) 155-162

15. Boulgouris, N., Plataniotis, K., Hatzinakos, D.: Gait recognition using linear time normalization. Pattern Recognition 39 (2006) 969-979

16. Mori, A., Makihara, Y., Yagi, Y.: Gait recognition using period-based phase synchronization for low frame-rate videos. In: Proc. of 20th Int. Conf. on Pattern Recognition, Istanbul, Turkey (2010)

17. Little, J., Boyd, J.: Recognizing people by their gait: The shape of motion. Videre: Journal of Computer Vision Research 1 (1998) 1-13

18. Makihara, Y., Sagawa, R., Mukaigawa, Y., Echigo, T., Yagi, Y.: Gait recognition using a view transformation model in the frequency domain. In: Proc. of the 9th European Conf. on Computer Vision. Volume 3., Graz, Austria (2006) 151-163

19. Veeraraghavan, A., Chellappa, R., Roy-Chowdhury, A.: The function space of an activity. In: Proc. of the 2006 IEEE Computer Society Conf. on Computer Vision and Pattern Recognition. Volume 1., New York, USA (2006) 959-966

20. Cuntoor, N., Kale, A., Chellappa, R.: Combining multiple evidences for gait recognition. In: Proc. of IEEE Int. Conf. on Acoustics, Speech, and Signal Processing. Volume 3. (2003) 33-36

21. Zhou, Z., Damper, R., Prugel-Bennett, A.: Model selection within a bayesian approach to extraction of walker motion. In: Proc. of the IEEE Computer Society Workshop on Biometrics 2006, New York, USA (2006)

22. Lee, L., Dalley, G., Tieu, K.: Learning pedestrian models for silhouette refinement. In: Proc. Int'l Conf. on Computer Vision 2003. Volume 1. (2003) 663-670

23. Liu, Z., Sarkar, S.: Effect of silhouette quality on hard problems in gait recognition. Trans. of Systems, Man, and Cybernetics Part B: Cybernetics 35 (2005) 170-183

24. Liu, Z., Sarkar, S.: Improved gait recognition by gait dynamics normalization. IEEE Transactions on Pattern Analysis and Machine Intelligence 28 (2006) 863876

25. Sunderesan, A., Chowdhury, A., Chellappa, R.: A hidden markov model based framework for recognition of humans from gait sequences. In: Proc. IEEE Int'l Conf. on Image Processing 2003. Volume 2. (2003) 93-96

26. Makihara, Y., Yagi, Y.: Silhouette extraction based on iterative spatio-temporal local color transformation and graph-cut segmentation. In: Proc. of the 19th Int. Conf. on Pattern Recognition, Tampa, Florida USA (2008) 